\title{
InteliMED - Proposta de Sistema de Apoio ao Diagnóstico Médico para Dispositivos Móveis
}

\author{
Júlio Menezes Jr. ${ }^{1}$, Cristine Gusmão ${ }^{1,2}$ \\ ${ }^{1}$ Grupo de Pesquisa S@BER: Tecnologias Educacionais e Sociais, \\ Universidade Federal de Pernambuco, Recife, Brasil \\ ${ }^{2}$ Programa de Pós-graduação em Engenharia Biomédica, Centro de \\ Tecnologia e Geociências (CTG), Universidade Federal de Pernambuco, \\ Recife, Brasil
}

\begin{abstract}
The structure of public health care in Brazil is organized in a way to provide decentralized services. In theory, this scenario would enable the usage of mobile devices to support primary health care. However, we realize that there is a gap in the usage of this technology in this domain. In addition, there is a need of decision-support tools that are based on collected evidences, once often the professional of primary health care is a generalist one, who may need information for supporting the decision-making process on more specific contexts. This paper proposes a decision-support system for medical diagnosis through computational intelligence and mobile devices (smartphones and tablets) - InteliMED.
\end{abstract}

Resumo. A estrutura de saúde pública no Brasil é organizada de modo a disponibilizar serviços de maneira descentralizada. Este cenário em tese viabilizaria o uso de dispositivos móveis para apoiar a atenção primária. Ainda assim percebe-se que há uma lacuna quanto ao uso desses meios em atenção primária à saúde, além de se fazerem necessárias ferramentas de apoio à tomada de decisão baseada em evidências coletadas, uma vez que muitas vezes o profissional de saúde, que essencialmente é generalista, sente a necessidade de um conjunto de informações que subsidiem a tomada de decisão em contextos mais especificos. Este artigo apresenta uma proposta de sistema de suporte à decisão para apoio ao diagnóstico médico utilizando inteligência computacional e dispositivos móveis (smartphones e tablets) InteliMED.

\section{Introdução}

Sem dúvida, a computação móvel é uma das tecnologias que se desenvolvem mais velozmente no mundo e no Brasil. Os dispositivos móveis estão cada vez mais presentes no nosso dia-a-dia, permitindo a transferência, muitas vezes rápida, de dados, sem haver a necessidade de uso de fios, através de tecnologias como Wi-Fi, Wi-Max, 3G, 4G e até mesmo Bluetooth. Outras grandes vantagens são o seu tamanho reduzido e leveza, permitindo que o dispositivo tenha o tamanho de um bolso de camisa e o custo que, a cada dia que passa, está mais acessível. Com todas estas vantagens e sua popularização, é um caminho natural a utilização da computação móvel também em sistemas de saúde. 
O uso de tecnologias móveis traz diversos benefícios quando utilizadas para auxílio da atenção primária à saúde. Alguns mais visíveis são: (i) simplificação do acesso e da atualização das informações; (ii) fortalecimento da medicina baseada em evidências através de sistemas de apoio à decisão; e (iii) diminuição de erros e inconsistências [Menezes Jr. et al. 2011].

Os serviços de atenção à saúde primária são, essencialmente, descentralizados e realizados por profissionais que possuem conhecimento generalizado, uma vez que o tipo de serviço é voltado para ações preventivas em comunidades. Em saúde comunitária, ou mais especificamente na Estratégia de Saúde da Família (ESF), há uma estrutura organizacional que viabiliza a utilização de dispositivos móveis, pois opera com unidades de saúde básicas distribuídas, nas quais as equipes de profissionais respondem por uma pequena parcela da população e, além disso, realizam visitas domiciliares [Menezes Jr. et al. 2011].

O uso de técnicas de inteligência computacional em sistemas que visam apoiar a decisão médica já é bastante comum [Kulikowski, Weiss 1982; Patil, Szolovits, Schwartz 1982; Shortliffe, Scott, Bischoff 1981; Razzouk, Shirakawa, Mari 2000; Seixas et al. 2011]. No contexto da atenção primária, percebe-se que esses sistemas de suporte à decisão também podem contribuir para a diminuição da ocorrência de casos graves e internações hospitalares realizados por um diagnóstico tardio.

Dentro deste contexto, este artigo apresenta a proposta de sistema de apoio à decisão descentralizado por meio do uso de dispositivos móveis - InteliMED [Gusmão, 2012; Menezes Jr. et al. 2012, Menezes Jr. et al. 2011]. O intuito deste trabalho é, não só apresentar o sistema proposto, mas também o seu processo de construção.

Após esta seção introdutória, o artigo está organizado da seguinte forma: a seção 2 traz a fundamentação teórica necessária ao entendimento da pesquisa; a seção 3 demonstra a solução proposta e suas peculiaridades; a seção 4 apresenta os resultados e contribuições obtidos; finalmente, a seção 6 descreve as considerações finais e trabalhos futuros.

\section{Fundamentação Teórica}

Esta seção apresenta os principais conceitos abordados para a concepção do InteliMED: Mobile Health, trabalhos relacionados e novas tecnologias móveis; Asma e sua relevância; uso de Inteligência Computacional para suporte ao diagnóstico médico; e uso simultâneo de diferentes metodologias ágeis de desenvolvimento de software.

\subsection{Mobile Health}

Mobile Health (ou mHealth) é o termo utilizado para a prática de medicina e saúde pública suportada por dispositivos móveis - tais como smartphones e tablets. A área de mHealth emergiu como um subsegmento de eHealth, que consiste no uso de Tecnologia da Informação e Comunicação para auxiliar serviços de saúde [Vital Wave Consulting 2009]. Aplicações mHealth podem incluir: o uso de dispositivos móveis na coleta de dados clínicos e de saúde da comunidade; o fornecimento de informações de saúde para os profissionais, pesquisadores e pacientes; e a prestação direta de cuidados via telemedicina móvel [Germanakos, Mourlas, Samaras 2005]. 
Conforme apresentado por Menezes Jr. e demais autores (2011), em uma análise comparativa entre sistemas móveis para saúde, observou-se que boa parte destes sistemas são disponibilizados para PDA (Personal Digital Assistant) e utilizam tecnologia J2ME (Plataforma Java 2 Micro Edition), a exemplo do HandMED [Castro et al. 2004] e do PDAEmbu [Moraes, Pisa, Lopes 2004].

Por outro lado, tecnologias para dispositivos móveis têm avançando com certa rapidez, devido à melhora considerável da capacidade de processamento e memória dos dispositivos. Assim, a construção de sistemas de software mais robustos para este tipo de dispositivo tornou-se tendência. Por exemplo, o Android (sistema operacional open source para dispositivos móveis desenvolvido pela Google e empresas parceiras), em se tratando apenas de smartphones, já é o sistema móvel mais utilizado no mundo [InfoGraphic Labs 2012].

\subsection{Asma}

A asma é uma das doenças crônicas mais comuns em todo o mundo. Segundo a Sociedade Brasileira de Pneumologia e Tisiologia [Sociedade Brasileira de Pneumologia e Tisiologia 2006], no Brasil a asma atinge cerca de $20 \%$ de crianças e adolescentes. Outro dado importante é que $2,3 \%$ de todas as internações pelo SUS (Sistema Único de Saúde) são por asma. Essas internações custam aproximadamente 76 milhões de reais, o que chega a ser o terceiro maior valor gasto com uma única doença no País.

A atenção básica à saúde desempenha uma importante função no diagnóstico e tratamento corretos de doenças, uma vez que é a principal porta de entrada dos pacientes no SUS através da ESF. O diagnóstico clínico da asma na atenção básica à saúde é importante para iniciar corretamente o tratamento, e consequentemente diminuir a ocorrência de casos graves e internações hospitalares com elevado custo. Deste modo, como prova de conceito, inicialmente optou-se pelo desenvolvimento de sistema voltado para o diagnóstico da Asma em crianças e adolescentes com idade entre 5 e 18 anos.

\subsection{Inteligência Computacional}

A mineração de dados - principal atividade do processo de descoberta de conhecimento (Knowledge Discovery in Databases - KDD) - consiste na aplicação de algoritmos com a finalidade de extrair conhecimento de bases de dados [Fayyad, Piatetsky-Shapiro, Smyth 1996]. Esta descoberta de conhecimento pode ser realizada com diversas técnicas, tais como: agrupamentos, hipóteses, regras de associação, árvores de decisão, redes neurais, redes bayesianas, dentre outras.

No caso do InteliMED, ao analisar seu contexto considerando o estudo realizado por Fayyad, Piatetsky-Shapiro e Smyth (1996), estamos lidando com um problema de classificação, pois o objetivo do sistema é classificar a partir das entradas (perguntas extraídas de questionário relacionado ao diagnóstico de asma) se o paciente possui ou não asma.

Conforme um estudo realizado por Menezes Jr. et al. (2011), a técnica de mineração de dados mais adequada para solução do problema tratado no InteliMED é árvore de decisão, uma vez que esta técnica possui grande aplicabilidade ao domínio do diagnóstico em saúde, devido à sua natureza de apoio a decisão baseada na inferência sobre dados coletados em paralelo com histórico de casos passados. Além disso, outra 
vantagem da árvore de decisão é que ela pode ser facilmente validada junto aos usuários do sistema, característica importante para o domínio de saúde, por tornar o uso da técnica um processo intuitivo.

As árvores de decisão são modelos estatísticos, que podem ser usados com o objetivo de aprender a classificar objetos. Neste tipo de aplicação, a partir de um conjunto de instâncias, cujas classes são conhecidas, após a fase de treinamento, uma árvore de decisão é capaz de classificar novas instâncias [Quinlan 1996]. Na Figura 1, pode-se observar um exemplo de árvore de decisão, onde estão ilustrados seus elementos [Ken et al. 2010]:

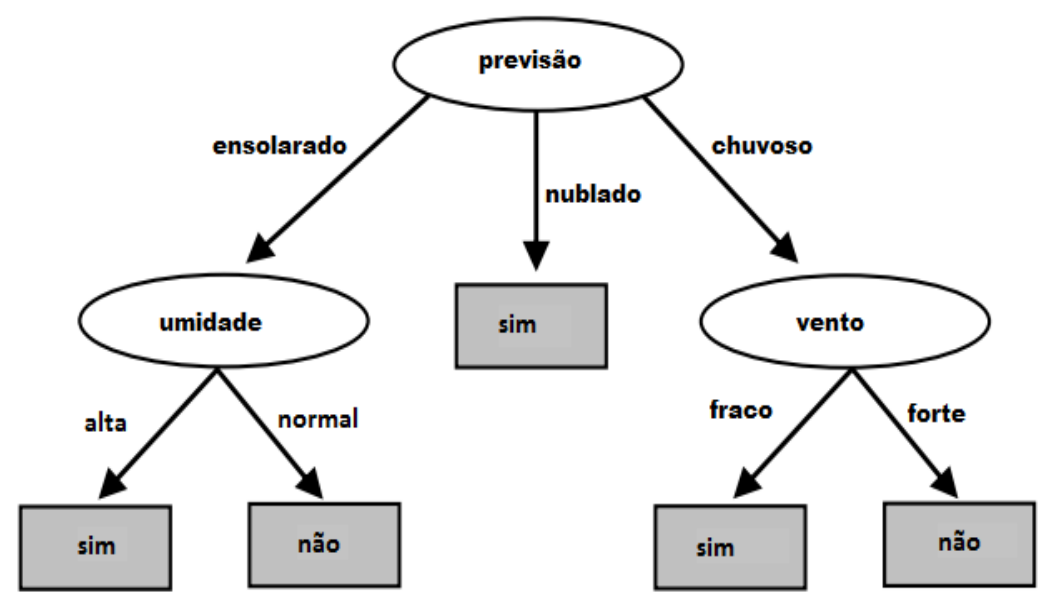

Figura 1. Exemplo de árvore de decisão adaptada [Ken et al. 2010]

De acordo com a Figura 1, para melhor entendimento da estrutura de uma árvore de decisão, tem-se os seguintes elementos:

1. Nós (elipses) - são os atributos que definem a classificação do objeto (previsão, umidade e vento), e no presente trabalho eles representam as perguntas de um questionário sobre asma;

2. Arestas (setas/testes) - definem os caminhos da árvore (Ex.: se previsão=chuvoso, então seguir para vento), e neste projeto as arestas representam as respostas às perguntas do questionário;

3. Folhas (retângulos) - indicam a classificação do objeto, que neste trabalho são restritas a: asma ou não asma.

\subsection{Metodologias Ágeis de Desenvolvimento de Software}

A aplicação de metodologias de desenvolvimento de software tradicionais, muitas vezes, produz software que não cumprem exigências de prazo, custos e escopo [Soares 2004] devido ao enfoque em itens que não agregam valor ao negócio, tais como o uso excessivo de documentação e processos pouco adaptativos. Neste cenário, surgiram as Metodologias Ágeis, que diferem das tradicionais, principalmente pelo enfoque em 
pessoas e em software funcionando desde o início, além de serem consideradas adaptativas [Beck et al. 2001].

As metodologias ágeis mais conhecidas atualmente são o XP (Extreme Programming) [Beck 1999] e o Scrum [Schwaber, Beedle 2002]. Elas possuem algumas semelhanças e diferenças que permitem que sejam utilizadas em conjunto, pois se complementam. Enquanto o Scrum foca principalmente em planejamento e gerência de projetos em geral, o XP possui enfoque em práticas de desenvolvimento de software.

O Scrum atribui papéis aos interessados no projeto, como stakeholders (todos os interessados), Scrum Master (responsável por garantir o fluxo do processo), Product Owner (representante do cliente) e Scrum Team (composto pela equipe de desenvolvimento multifuncional e autogerenciável, pelo representante do cliente e pelo Scrum Master). O Scrum possui alguns artefatos, dentre os quais se destacam: Burn Down Chart é um gráfico que representa a quantidade de esforço que falta para a conclusão das tarefas estipuladas para um determinado tempo; Product Backlog é uma lista priorizada de requisitos de alto nível; e Sprint Backlog é uma lista de funcionalidades a serem implementadas na iteração.

O XP sugere a aplicação da técnica de programação em pares, o que acelera o desenvolvimento e diminui a probabilidade de erros, visto que mais de um programador trabalha em um código ao mesmo tempo. Esta técnica também é útil em casos de evasão, já que o conhecimento acerca dos códigos do sistema está distribuído entre os programadores - propriedade coletiva. Além disso, elaboração de estórias do usuário, refatoração de código, integração contínua, testes e prototipação são práticas comuns em projetos que utilizam essa metodologia.

Apesar das peculiaridades de cada processo, existem também várias práticas que são comuns entre Scrum e XP. O Scrum divide o desenvolvimento em Sprints (de 2 a 4 semanas), o que pode ser comparado a cada iteração do XP. Reuniões de planejamento a cada iteração são comuns a ambos os processos, bem como uma reunião curta (com os nomes de Daily Scrum, no Scrum e Daily Meeting, no XP) feita diariamente. Nessas reuniões diárias são discutidos: o que falta ser feito até o término da iteração; as dificuldades encontradas; e os avanços desde a última reunião de iteração. Além do mais, ambas as metodologias prezam pelo cliente inserido no processo de desenvolvimento.

Para melhor entendimento da proposta apresentada a seguir, os conceitos apresentados nesta seção são de fundamental importância para a justificativa das ações tomadas ao longo do projeto. Deste modo, o restante do artigo descreve a aplicação de metodologias ágeis no desenvolvimento, do projeto, que utilizou técnica de inteligência computacional para suporte a decisão utilizando dispositivos móveis - Tablets e Smartphones.

\section{InteliMED ${ }^{1}$}

Nesta seção, são apresentados: a metodologia, incluindo técnicas e ferramentas, empregada durante o planejamento e construção do InteliMED; o funcionamento do

\footnotetext{
${ }^{1}$ InteliMED na web: http://promise.cin.ufpe.br/mhealth e http://promise.cin.ufpe.br/intelimed
}

MENEZES JR., J.; GUSMAO, C. InteliMED - Proposta de Sistema de Apoio ao Diagnóstico Médico para Dispositivos Móveis. iSys - Revista Brasileira de Sistemas de Informação, Rio de Janeiro, vol. 6, No. 1, p. 44-61, 2013. 
sistema, bem como tecnologias e arquitetura utilizadas; e o processo de descoberta de conhecimento em base de dados.

\subsection{Processo de Desenvolvimento}

Devido a multidisciplinaridade do sistema, o InteliMED foi desenvolvido por equipes compostas por profissionais e estudantes de saúde e de computação. A equipe de saúde auxiliou na coleta e validação de dados, bem como na elaboração do questionário utilizado como entrada para o algoritmo inteligente. Os questionários foram aplicados a pacientes atendidos em serviços ambulatoriais de alergia e de pediatria e adolescência. A equipe de computação, por sua vez, planejou e desenvolveu todo o sistema computacional.

Durante o processo de desenvolvimento do InteliMED a metodologia aplicada foi considerada híbrida, possuindo características adaptadas principalmente de dois métodos ágeis - o XP e o Scrum. A escolha por uma metodologia híbrida partiu do pressuposto de que o processo de desenvolvimento é instanciado de acordo com as características do projeto e da equipe, que neste caso é reduzida e por se tratar de um projeto cuja documentação técnica - requisitos e arquitetura - se fez necessária para justificar a aplicação do seu orçamento como um dos artefatos de software. Assim, o processo resultante foi iterativo e incremental, já que diversas partes do sistema eram desenvolvidas ao mesmo tempo e a cada iteração era concluída uma parte do sistema funcionando [Sommerville 2004].

Dispositivos móveis possuem diversos problemas quanto à interação com o usuário, dentre outros motivos por possuírem tamanhos de telas pequenos e diferentes dos que o usuário está habituado. Assim, foi feita prototipação de telas baseada na apresentação Excellence in the Android User Experience [Nurik 2010] do Google Developer Day 2010 aprovada junto ao Product Owner - um estudante de medicina vinculado ao projeto.

Além de prototipação, aplicou-se programação em pares, refatoração de código e integração contínua provenientes do XP. Estórias de Usuários e um Product Backlog foram produzidos pelo Scrum Team, principalmente pelo Scrum Master e pelo Product Owner. Sprint Backlogs foram estimados utilizando técnica de Planning Poker, que permite que os próprios programadores estimem quanto tempo levam para completar uma determinada tarefa.

A implementação do módulo servidor foi integrada ao Jenkins, sistema de gerenciamento com integração contínua que possui diversas funcionalidades para auxiliar o monitoramento e a execução automática de testes [Smart 2011], e ao Sonar, plataforma de gestão da qualidade dedicada a analisar e medir continuamente a qualidade do código fonte [SonarSource 2011]. Além disso, o sistema móvel também foi integrado a um projeto responsável por todos os testes unitários, através do framework de testes jUnit para Android [Google Inc. 2010].

Com o objetivo de intensificar a comunicação entre as equipes, foram utilizadas algumas ferramentas, tais como Yammer [Yammer 2012], Manymoon [Manymoon 2011], Google Groups [Google Inc. 2012], Skype [Skype 2012] e Team Viewer [Team Viewer 2012]. O Yammer, que é uma rede social corporativa, auxiliou o grupo quanto a troca de informações que exigiam comunicação rápida. No Manymoon, que auxiliou no 
gerenciamento de atividades, eram cadastradas tarefas com seus respectivos prazos. Cada pessoa do grupo escolhia uma tarefa a fazer, sendo possível, por exemplo, saber qual programador implementou qual funcionalidade e em quanto tempo. Informações de interesse de todas as equipes eram compartilhadas no grupo de discussão do Google. Para reuniões que não podiam ser presenciais, utilizou-se o Skype, para compartilhamento de mensagens de áudio entre todos os integrantes, em conjunto com o Team Viewer, no qual um membro compartilhava a tela com todos os demais.

Devido a equipe de desenvolvimento ser constituída por mais de um programador, houve a necessidade de gerenciar o acesso a arquivos compartilhados. Para tal, foi utilizado o sistema de controle de versão SVN [CollabNet 2009], por ser do modo Copy-Modify-Merge, o que permite que mais de uma pessoa trabalhe em um arquivo ao mesmo tempo. O código de todo o projeto está integrado com um repositório no Google Code [Google Inc. 2011].

\subsection{Proposta do InteliMED - Funcionalidades e Arquitetura}

O sistema é composto por dois módulos: aplicação móvel e servidor. Dois times trabalharam nesses módulos, ambos de computação. A arquitetura do InteliMED pode ser vista na Figura 2. A visão global da arquitetura mostra que a aplicação está distribuída entre o equipamento móvel e o servidor.

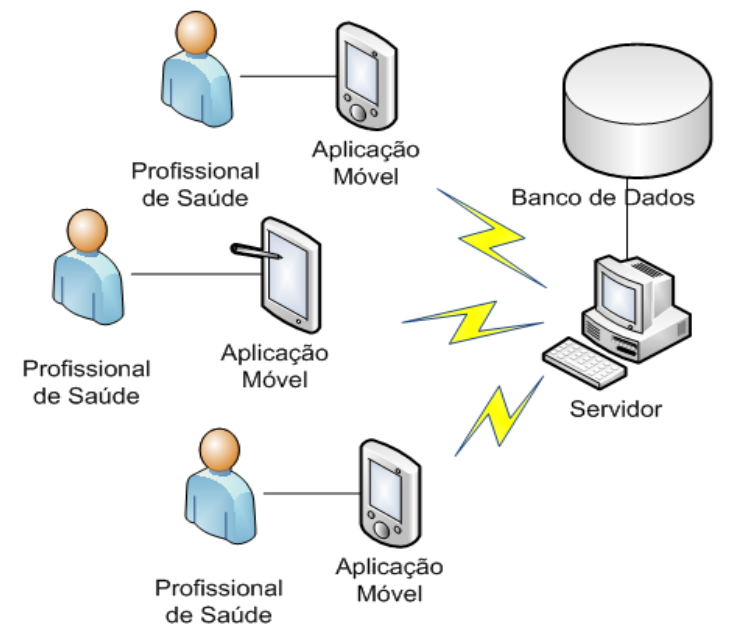

Figura 2. Arquitetura do InteliMED

A aplicação foi desenvolvida utilizando a linguagem Java na plataforma Android, versão 2.2. A principal funcionalidade do InteliMED é prover o diagnóstico de asma, que inclusive é executada mesmo sem conexão com o servidor, aumentando a aplicabilidade prática do sistema, pois possibilita seu uso em áreas onde a rede é limitada de recursos, ou até mesmo inexistente. Esta decisão de projeto é pertinente, uma vez que as Unidades de Saúde da Família normalmente atendem a comunidades carentes.

A funcionalidade da aplicação móvel que determina o diagnóstico ocorre quando o médico responde a um questionário exibido na tela, coletando evidências, as quais serão utilizadas pela aplicação móvel para determinar o diagnóstico. A árvore de decisão é percorrida de acordo com as respostas do questionário e, concluído o processamento, o resultado é informado ao usuário. Após exibir o diagnóstico, o aplicativo questiona se o médico concorda com o resultado, ou não, e possibilita a inserção de comentários 
adicionais. Todas as respostas são armazenadas em banco de dados do próprio dispositivo (SQLite) para posterior sincronização com o servidor.

Outras funcionalidades do sistema móvel são:

- "Enviar Evidência" - tem o objetivo de enviar as informações do diagnóstico (incluindo opinião médica), que estão armazenadas localmente, para o servidor. Este envio é realizado via conexão HTTP utilizando REST (REpresentational State Transfer).

- "Atualizar Árvore" - possibilita a atualização da árvore de decisão do dispositivo móvel, a antiga é removida e usa-se a gerada pelo servidor.

Já o servidor foi concebido para armazenar a árvore de decisão para diagnóstico de asma, resultante da aplicação do algoritmo de mineração de dados C4.5, que é uma implementação da técnica de árvore de decisão, conforme detalhado na seção 3.3 [Quinlan 1992]. A ideia é que o servidor seja executado dentro de uma Unidade de Saúde Básica, possibilitando que o script inteligente represente a comunidade atendida pela Unidade, já que o mesmo é gerado a partir de casos passados. A Figura 3 apresenta a captura de tela do servidor. Através desta aplicação, o usuário pode visualizar a estrutura da árvore de decisão, bem como os resultados capturados dos dispositivos móveis.

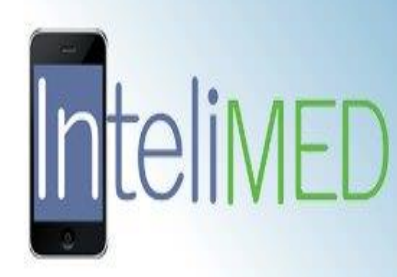

\begin{tabular}{|c|c|}
\hline \multicolumn{2}{|c|}{ As Home $f_{0}$ Novo(a) No } \\
\hline \multicolumn{2}{|c|}{ No Lista } \\
\hline Id & Descricao \\
\hline 1 & Seu filho ou sua filha tosse ou tem chiado no peito mesmo quando nao esta resfriado? \\
\hline 2 & Seu filho ou sua filha tem crises de tosse ou chiado no peito quando tem contato com poeira de casa? \\
\hline 3 & Nos ultimos 12 (doze) meses, quantas crises de sibilos (chiado no peito) seu filho(a) teve? \\
\hline 4 & Seu filho ou sua filha tosse ou tem chiado no peito quando se acorda? \\
\hline 5 & Seu filho(a) tem crises de tosse ou sibilos (chiado no peito) quando faz exercicios fisicos, do tipo correr ou jogar bola? \\
\hline 6 & Seu filho ou sua filha vem apresentando aperto no peito ou dificuldade para respirar? \\
\hline 7 & Seu filho(a) melhora da tosse ou chiado quando usa sprays, bombinhas ou nebulizacoes? \\
\hline 8 & Seu filho(a) tem crises de tosse ou chiado no peito quando tem contato com mofo? \\
\hline 9 & Asma \\
\hline 10 & Nao asma \\
\hline 12 & Próximo \\
\hline
\end{tabular}

\section{Figura 3. Tela do Servidor}

A tecnologia utilizada para implementação do servidor foi Grails, um framework para construção de aplicações web através da linguagem de programação 
Groovy (uma linguagem dinâmica para a plataforma Java) com foco em alta produtividade e desenvolvimento rápido de aplicações web [SpringSource 2011]. Ao receber os dados do dispositivo móvel por meio de REST, o servidor automaticamente os armazena em seu banco de dados (MySQL) através do framework. No servidor, são armazenadas as evidências enviadas pela aplicação móvel, com o objetivo de melhorar a árvore de decisão, por meio da utilização dos dados coletados.

Na perspectiva lógica, na realidade existem duas arquiteturas distintas: uma para o equipamento móvel e outra para o servidor. Estas arquiteturas se comunicam entre si através do protocolo HTTP.

A Figura 4 apresenta a arquitetura da aplicação móvel. Este modelo contém três componentes principais:

1. Database: responsável por armazenar árvore recebida do servidor e evidências coletadas a partir de diagnósticos da própria aplicação móvel;

2. Diagnostic: componente responsável por exibir questionário, percorrer a árvore e exibir diagnóstico, bem como coletar evidência a partir de opinião médica;

3. Communication: responsável pelo envio e recebimento de mensagens entre o equipamento móvel e servidor.

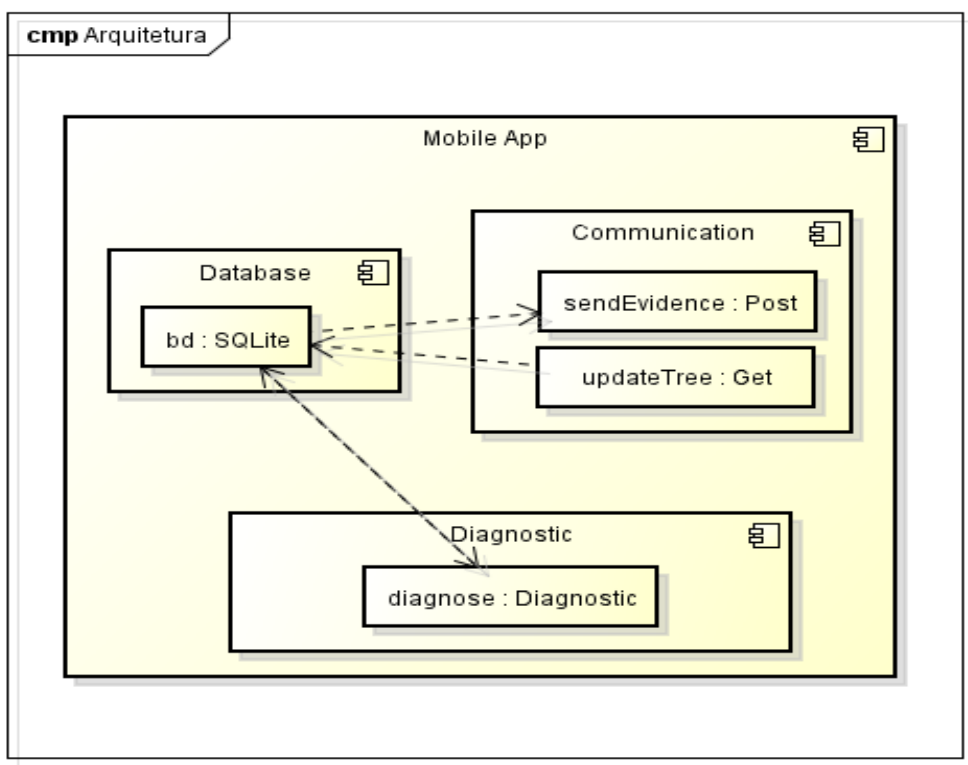

Figura 4. Visão da Arquitetura Equipamento Móvel

A Figura 5 apresenta a arquitetura do servidor. Esta arquitetura é baseada em servidores de aplicação web e é composto pelos seguintes componentes:

1. Controller: responsável por tratar requisições passadas pelo dispositivo móvel ou servidor inteligente ao servidor;

2. Domain: responsável por manter as entidades relacionadas ao domínio da aplicação. 


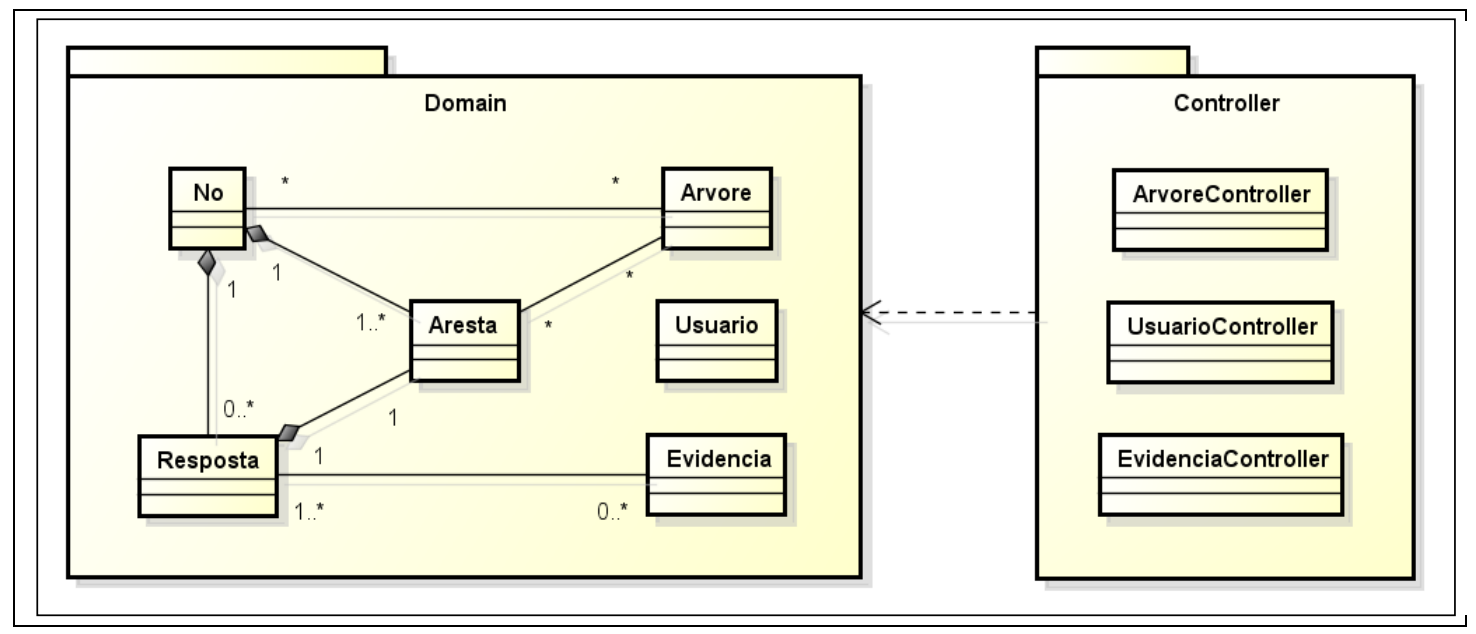

Figura 5. Visão da Arquitetura do Servidor

Um aspecto importante a ser destacado no componente Domain é a representação da estrutura da árvore de decisão em banco de dados de modo a favorecer a escalabilidade do InteliMED. Assim, de fato, o servidor apresenta um modelo de uma árvore de decisão em banco de dados, o que facilita a alteração de dados e informações, bem como o uso de outros tipos de questionários baseados em árvores de decisão.

\subsubsection{Fluxo de Informação}

A Figura 6 apresenta o fluxo de telas mais comum no uso do InteliMED. Na Figura 6(A) o usuário, que no caso é representado pelo profissional médico, é validado através da inserção de nome de usuário e senha.

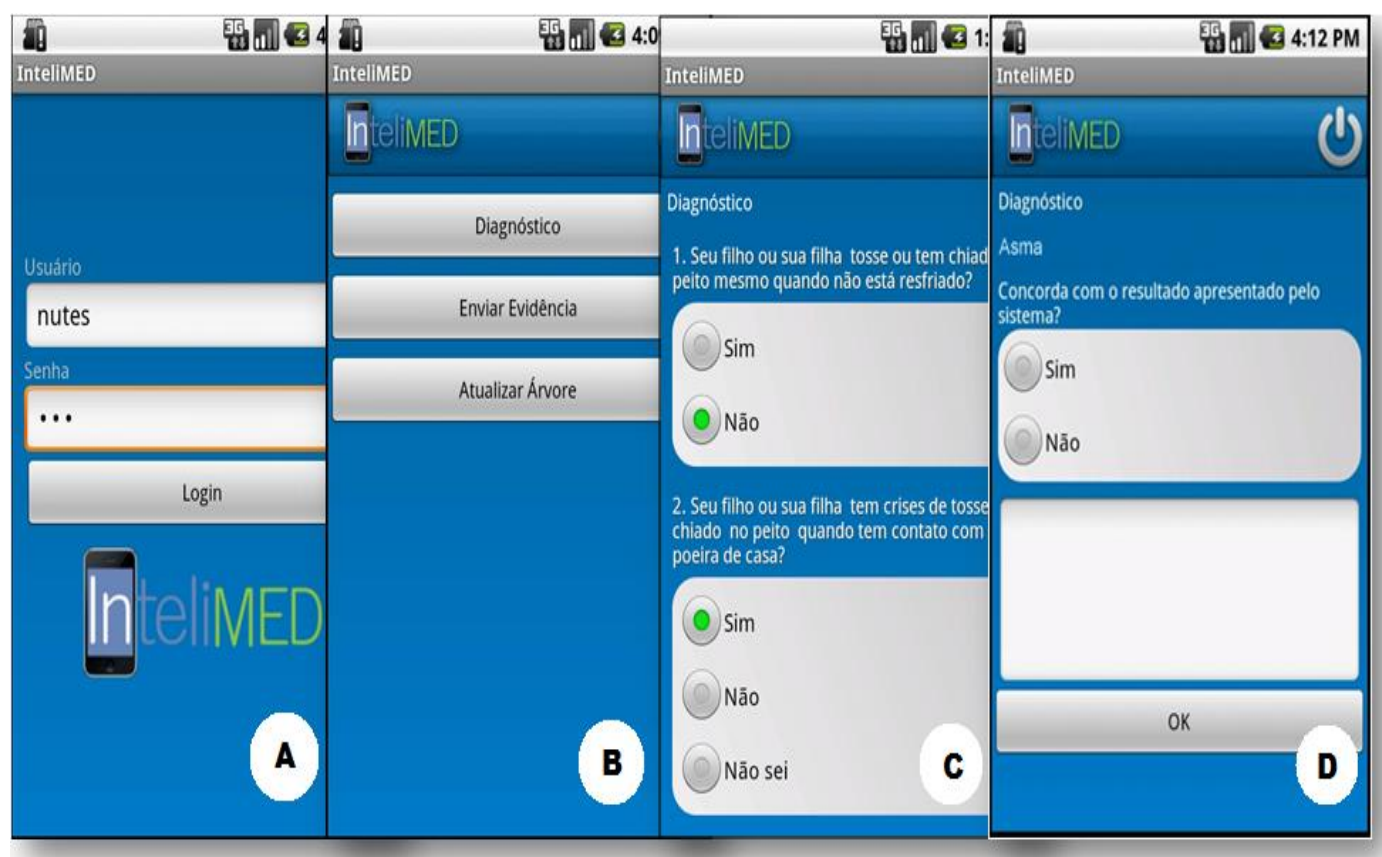

Figura 6. Telas do sistema móvel

MENEZES JR., J.; GUSMAO, C. InteliMED - Proposta de Sistema de Apoio ao Diagnóstico Médico para Dispositivos Móveis. iSys - Revista Brasileira de Sistemas de Informação, Rio de Janeiro, vol. 6, No. 1, p. 44-61, 2013. 
Ao acessar o sistema o usuário tem acesso ao menu principal, no qual ele pode diagnosticar, enviar evidências e atualizar o script da árvore utilizada nos diagnósticos, como ilustrado na Figura 6(B). Ao selecionar a opção "Enviar evidência" ou "Atualizar árvore", é exibida uma mensagem enquanto os dados são sincronizados, e vale ressaltar que estas duas últimas funcionalidades requerem conexão à internet para sua concretização.

Já ao selecionar a opção "Diagnóstico", o sistema irá apresentar um questionário, conforme pode ser visto na Figura 6(C). Após o preenchimento do questionário é exibida a tela com o resultado processado pelo sistema, e o usuário neste momento deve informar se concorda com o resultado, bem como justificar o motivo da concordância ou não, como pode ser visto na Figura 6(D).

\subsection{Processo de Mineração de Dados}

A mineração de dados - principal atividade do processo de descoberta de conhecimento (Knowledge Discovery in Databases - KDD) - consiste em aplicar algoritmos com a finalidade de extrair conhecimento de bases de dados [Fayyad, Piatetsky-Shapiro, Smyth 1996]. Neste projeto, conforme justificado na seção 2, o algoritmo escolhido foi árvore de decisão. Para auxiliar o processo de KDD foi utilizada uma ferramenta de código aberto chamada WEKA (Waikato Environment for Knowledge Analysis), que possui implementações de vários algoritmos de mineração de dados [Hall et al. 2009].

Como entradas para o algoritmo foram utilizados dados obtidos a partir de um questionário com 40 questões pré-selecionadas pela equipe de saúde. Este questionário inicialmente aplicado é uma adaptação do ISAAC ("International Study of Asthma and Allergies in Childhood") [Solé et al. 1998], que é modelo de referência mundial para o apoio ao diagnóstico e tratamento de asma em crianças e adolescentes.

Foram realizados 34 experimentos utilizando a ferramenta WEKA. Devido ao número reduzido de dados coletados (apenas 93 pacientes responderam os questionários), houve a necessidade da criação de mais instâncias. Desta forma, com o auxílio da equipe de saúde, especialistas no domínio da asma, foram criados mais 50 cenários simulados, o que totalizou 143 instâncias, um número ainda pequeno, porém suficiente para resultar numa árvore com taxa de acerto de 91,61\%. Esta taxa de acerto pode ser considerada aceitável, devido a natureza de apoio a decisão do sistema, ou seja, o sistema visa auxiliar o médico no diagnóstico e não substituí-lo nesta tarefa.

O algoritmo J48, que é uma implementação da técnica para construção de árvore decisão conhecida como C4.5, foi utilizado em todos os experimentos, com seed $=1313$ e sem a primeira pergunta do questionário de 40 perguntas. A primeira pergunta foi removida em comum acordo com a equipe de saúde, pois ela já está contemplada na segunda pergunta do questionário. Além disso, variou-se automaticamente os valores do minNumObj[2;3;4;5] e confidenceFactor $[0.1 ; 0.2 ; 0.3 ; 0.4 ; 0.5]$ para obter o melhor resultado. A seguir são descritas as variações que foram utilizadas nos experimentos [Quinlan 1992]:

- V1: Poda - o algoritmo foi executado com a opção de poda ativa;

- V2: Sem poda - o algoritmo foi executado com a opção de poda desativada; 
- V3: Pré-processamento 1 - antes da execução do algoritmo foi feita a seleção de atributos utilizando o algoritmo geneticSearch;

- V4: Sem pré-processamento - a execução do algoritmo foi realizada sem nenhum tipo de pré-processamento.

- V5: Pré-processamento 2 - antes da execução do algoritmo foi feita a seleção de atributos utilizando as 20 questões mais importantes de acordo com a opinião de especialistas da área de saúde.

- V6: Pré-processamento 3 - antes da execução do algoritmo foi removido o atributo 36 ( $36^{\mathrm{a}}$ pergunta do questionário), pois estava enviesando o resultado final.

Após a realização dos experimentos, chegou-se à conclusão de que a melhor combinação foi uma instância que combinava V1 com V5, pois apresentou melhor taxa de acerto e foi validada com especialistas no domínio do diagnóstico da asma em crianças e adolescentes.

\section{Contribuições}

Além do próprio sistema, diversos artefatos foram produzidos, a partir de testes e avaliação junto ao time de saúde. Também podemos destacar o alto impacto social do projeto, pois fomenta a produção de inovação tecnológica para o domínio da atenção primária, que ainda carece de melhorias. Esta seção apresenta a árvore de decisão e os testes e avaliações realizados.

\section{1. Árvore de Decisão}

A construção da árvore de decisão gerada a partir do processo de mineração de dados aplicado aos dados obtidos pela equipe de saúde foi outro relevante artefato gerado. A Figura 7 representa a árvore de decisão escolhida para implementação no sistema. 


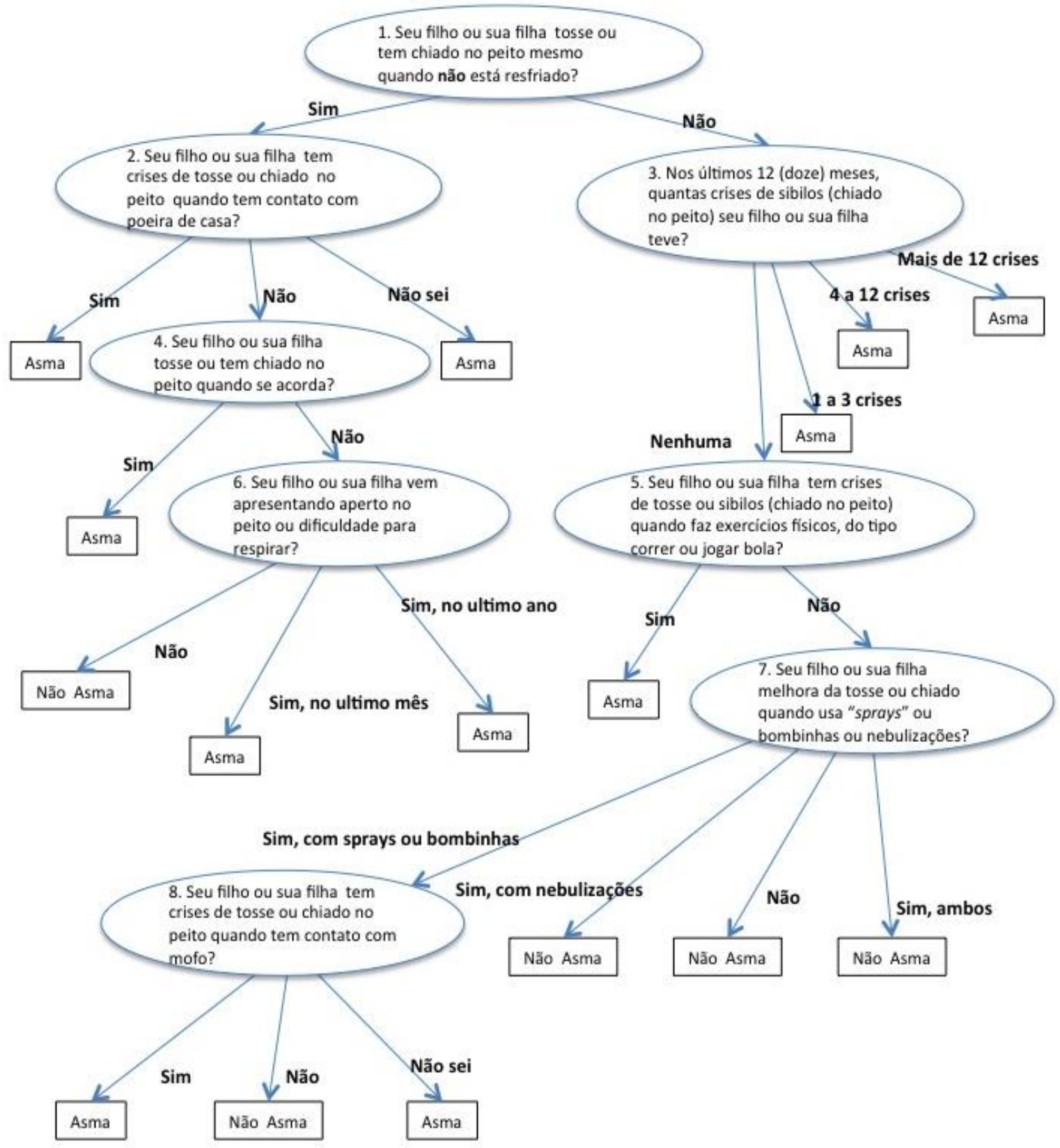

Figura 7. Árvore de decisão definida

A árvore foi gerada utilizando inicialmente guidelines de saúde, com o apoio de especialistas, e o algoritmo $\mathrm{C} 4.5$, de modo a torná-la sucinta, rápida, mas com capacidade de classificação de aceitável. Desta forma, como observado na Figura 7, o objetivo foi alcançado, pois o questionário inicialmente utilizado possuía 40 perguntas e ao final da execução do algoritmo, este ficou reduzido a apenas 8 perguntas, que em tese apoiam o diagnóstico de Asma para crianças e adolescentes com idade entre 5 e 18 anos de maneira satisfatória.

\subsection{Testes}

Foram elaborados planos de testes através de casos de testes, que especificavam descrição, pré-condições e os resultados esperados do processamento. Foram feitos ao 
todo 44 casos de testes funcionais, sendo 7 realizados por desenvolvedores e 37 por usuários com domínio da área médica. O sistema também foi testado por médicos sem vínculos com o projeto, em unidades de saúde básica.

Os testes de sistema, realizados por desenvolvedores, avaliaram o funcionamento do sistema no ambiente de produção de maneira integrada, e as execuções dos planos de testes duraram em média 60 minutos. Nesses testes, além de utilizar o equipamento móvel foi necessário acessar o servidor para checar a integridade de todo o sistema.

Já os testes executados por usuários com domínio da área médica focaram nos requisitos específicos do servidor e da aplicação móvel. Em média cada execução de plano de teste teve duração de 20 minutos. Os principais casos de testes funcionais executados foram: 1) Efetuar login - com dados corretos, incorretos e campos vazios; 2) Realizar Diagnóstico - um teste para cada um dos 16 possíveis caminhos da árvore; 3 ) Armazenar Evidência - testes nos quais o médico concorda ou não com o resultado, além de justificar sua opinião; 4) Enviar evidência para o servidor - testes que possuíam bases de dados contendo evidências em que o médico concordou com o resultado e contendo evidências em que o médico não concordou com o resultado e justificou; 5) Atualizar árvore de decisão através do servidor para classificação de asma - testes com a base de dados local contendo uma árvore pré-cadastrada para sugerir a uma pessoa ir ou não jogar tênis e contendo uma árvore pré-cadastrada para classificação de asma. 6) Inserção de novo usuário; 7) Editar informações de usuário cadastrado; e 8) Exclusão de usuário.

Os testes de sistema realizados foram criados pela permutação dos itens 2 , 4 e 5 da lista de casos de testes funcionais realizados, e foi realizado um teste adicional devido a sua importância: atualizou-se a árvore de decisão e depois testou-se todos os 16 possíveis caminhos da árvore de decisão.

Adicionalmente, foi realizada avaliação de usabilidade através de teste com usuários e avaliação heurística, pela qual foram avaliadas 24 heurísticas de cores, conteúdo, imagens, instruções, navegação e usabilidade, das quais 16 atendem completamente as recomendações, 7 atendem parcialmente e apenas uma, que exige que todos os botões e textos habilitados tenham textos de ajuda, não atende completamente. Nos testes com utilizadores, foram passadas algumas tarefas, tais como: obter diagnóstico de um paciente. Observou-se que usuários de smartphones, mesmo que sem sistema Android, executavam mais rapidamente as tarefas. Os utilizadores sugeriram algumas melhorias, como: (i) quando o sistema entrar em "descanso" seria interessante armazenar o estado do sistema no qual o usuário se encontra, para que ao retornar do "descanso" ele possa ir para a tela de autenticação e posteriormente possa voltar ao estado salvo; (ii) no menu iniciar, ao clicar sobre o botão "voltar" do dispositivo, seria bom que o sistema, não apenas fizesse logoff, mas também fechasse a aplicação.

\subsection{Avaliação Preliminar em Ambiente Real}

Atualmente o InteliMED vem sendo utilizado no ambulatório de alergia do Hospital das Clínicas da Universidade Federal de Pernambuco (HC/UFPE) para fins de pesquisa e avaliação quanto ao uso e à taxa de acerto da árvore de decisão. Este processo vem sendo aplicado com o apoio de estudantes e médicos residentes, através do uso de tablet (modelo Motorola Xoom), no qual o profissional ou o médico residente, para cada 
paciente, preenche o questionário do InteliMED, que informará se o paciente tem asma ou não. Ao final, o médico deve informar se concorda com o resultado informado pelo InteliMED.

Apesar da proposta do InteliMED ser para atenção primária, neste primeiro momento optou-se por aplicar o sistema em um cenário da esfera terciária (hospital) em um setor onde há diversos casos de doenças respiratórias, pois o objetivo inicialmente é realizar análise do ponto de vista de especialistas no domínio com o intuito de identificar pontos de melhoria.

A Tabela 1 a seguir apresenta os resultados preliminares. Até o momento o sistema foi testado em nove pacientes, selecionados de maneira aleatória. Quatro eram não asmáticos e cinco eram asmáticos, e o InteliMED acertou em oito oportunidades, somente tendo errado no Paciente 6, no qual o sistema apontou um falso positivo.

Tabela 1. Resultados preliminares por paciente

\begin{tabular}{|l|l|l|}
\hline Paciente & Resultado & Concorda com o resultado? \\
\hline 1 & Não tem asma & Sim \\
\hline 2 & Não tem asma & Sim \\
\hline 3 & Asma & Sim \\
\hline 4 & Asma & Sim \\
\hline 5 & Asma & Sim \\
\hline 6 & Asma & Não \\
\hline 7 & Asma & Sim \\
\hline 8 & Asma & Sim \\
\hline 9 & Não tem asma & Sim \\
\hline
\end{tabular}

Apesar de a amostra ainda ser bastante reduzida, o sistema acertou na grande maioria dos casos, e vem tendo boa aceitação por parte dos usuários. Outro ponto a destacar é a facilidade e rapidez do uso, uma vez que o sistema não gerou sobrecarga de trabalho e de tempo nos atendimentos realizados, tampouco apresentou erros decorridos por falhas (bugs) do sistema.

\section{Considerações Finais e Trabalhos Futuros}

Este trabalho apresentou conhecimentos aplicados na experiência de construção de um sistema de apoio a decisão médica desenvolvido como prova de conceito, bem como a proposta em si. Técnicas de mineração de dados foram aplicadas, produzindo uma árvore de decisão capaz de sugerir um diagnóstico (com taxa de acerto de 91,61\%) para asma.

Dentre as dificuldades encontradas durante o desenvolvimento deste trabalho algumas devem ser destacadas: a dificuldade de obtenção de equipamentos como smartphones e tablets para os testes; a integração de equipes distribuídas fisicamente, parcialmente resolvida com uso de ferramentas de apoio; evasão de desenvolvedor, problema pequeno devido à aplicação do conceito de propriedade coletiva. 
Atualmente o InteliMED continua sendo aplicado no ambulatório de alergia do Hospital das Clínicas da UFPE, visando aumentar o número de pacientes atendidos, possibilitando uma análise mais bem fundamentada dos resultados.

Como trabalhos futuros, planeja-se que o InteliMED seja implantado em um servidor usando o conceito de computação nas nuvens, de modo a prover maior facilidade na implantação prática do sistema. Pretende-se também a adaptação da arquitetura do InteliMED de modo a prover ações de educação por meio de guidelines em comunidades assentamentos rurais. Por fim, pretende-se utilizar o sistema em unidades de saúde da família com o objetivo de avaliar o sistema no contexto da atenção primária à saúde.

\section{Agradecimentos}

Os autores agradecem à FINEP (Financiadora de Estudos e Projetos) e ao CNPq (Conselho Nacional de Desenvolvimento Científico e Tecnológico) pelo apoio financeiro recebido para a execução deste projeto.

\section{Observações Adicionais}

Todos os procedimentos de coleta de dados de pacientes foram realizados após aprovação do Comitê de Ética em Pesquisas com Seres Humanos do Hospital Universitário Osvaldo Cruz sob o número de protocolo 100/2010.

\section{Referências}

Beck, K. (1999). "Programação Extrema Explicada". Bookman.

Beck, K. et al. (2001). "Agile Manifesto". Disponível em: <http://agilemanifesto.org/>. Acessado em Dezembro de 2011.

Castro, L.S.S. et al. (2004). "HandMed - Um Sistema Móvel Integrado para Captura Automática de Sintomas". In: IX Congresso Brasileiro de Informática na Saúde.

CollabNet. (2009). “Tigris Subversion”. Disponível em: <http://subversion.tigris.org/> Acessado em Dezembro de 2011.

Fayyad, U., Piatetsky-Shapiro, G., Smyth, P. (1996). "From data mining to knowledge discovery in databases". AI Magazine, v. 17, p. 37-54.

Germanakos, P., Mourlas, C., \& Samaras, G. (2005). "A Mobile Agent Approach for Ubiquitous and Personalized eHealth Information Systems". Proceedings of the Workshop on 'Personalization for e-Health' of the 10th International.

Google Inc. (2010) “Android Developers Guide - Testing Fundamentals - jUnit". Disponível em: $<$ http://developer.android.com/guide/topics/testing/testing_android.html\#JUnit>. Acessado em Dezembro de 2011.

Google Inc. (2012). “Google Groups”. Disponível em: <https:/groups.google.com>. Acessado em Janeiro de 2012.

Google Inc. (2011). “Google Code”. Disponível em: <http://code.google.com/int1//> Acessado em Dezembro de 2011. 
Gusmão, C. M. G. (2012). "Relatório Técnico FINEP - Suporte Remoto a Diagnóstico Médico utilizando Tecnologias Inteligentes”. Relatório Técnico. Janeiro de 2012.

Hall, M. et al. (2009). "The weka data mining software: An update". SIGKDD Explorations, v. 11.

InfoGraphic Labs. (2012). "Android vs iPhone". Disponível em: $<$ http://infographiclabs.com/infographic/best-of-the-smartphones-android-vs-iphone4/>. Acessado em Janeiro de 2012.

Ken, F. et al. A Tree-Based Decision Model to Support Prediction of the Severity of Asthma Exacerbations in Children. Journal of Medical Systems, v.34, p.551-562, 2010 .

Kulikowski, C. A., Weiss, S. (1982). "Representation of expert knowledge for consultation. The CASNET and EXPERT projects". In: Szolovitz, P. (Ed.) Artificial Intelligence in Medicine. Westview Press, Boulder, CO, pp. 21-55.

Manymoon. (2011) "Manymoon". Disponível em: <https://manymoon.com/>. Acessado em Dezembro de 2011.

Menezes Jr., J. et al. (2012) Mobile System for Decision Support in Medical Diagnosis: An Experience Report. In: 17th Annual International Meeting and Exposition of the American Telemedicine Association, 2012, San Jose, Estados Unidos. Abstracts from The American Telemedicine Association Seventeenth Annual International Meeting and Exposition, 2012. v. 18. p. 113-113.

Menezes Jr., J. et al. (2011). "InteliMED: uma experiência de desenvolvimento de sistema móvel de suporte ao diagnóstico médico". Revista Brasileira de Computação Aplicada. Passo Fundo, v. 3, n. 1, p. 30-42.

Moraes, D. A., Pisa, I. T., Lopes, P. R. L. (2004). "Protótipo para Coleta de Informações em Saúde Utilizando Dispositivos Móveis". In: IX Congresso Brasileiro de Informática na Saúde.

Nurik, R. (2010). "Excellence in the Android User Experience". Disponível em: $<$ http://www.slideshare.net/mobilegui/excellence-in-the-android-user-experience $>$. Acessado em Setembro de 2011.

Patil, R. S., Szolovits, P., Schwartz, W. B. (1982). "Modeling knowledge of the patient in acid-base and electrolyte disorders". In: Szolovitz, P. (Ed.) Artificial Intelligence in Medicine. Westview Press, Boulder, CO, pp. 191-226.

Quinlan, J. (1996). Learning decision tree classifiers. ACMComputing Surveys (CSUR), 28(1):71-72.

Quinlan, J. (1992). C4.5: Programs for Machine Learning. Morgan Kaufmann.

Razzouk, D., Shirakawa, I., Mari, J. de J. (2000). "Sistemas inteligentes no diagnóstico da esquizofrenia". Revista Brasileira de Psiquiatria [online]. Vol.22, suppl.1, pp. 3537. Disponível em: <http://www.scielo.br/pdf/rbp/v22s1/a12v22s1.pdf $>$ Acessado em Dezembro de 2011.

Schwaber, K., Beedle, M. (2002). "Agile Software Development with SCRUM". Prentice-Hall. 
Seixas, F. L. et al. (2011). "Sistema de Apoio à Decisão Aplicado ao Diagnóstico da Doença de Alzheimer". XI Workshop de Informática Médica. XXXI Congresso da Sociedade Brasileira de Computação. Disponível em: $<$ http://www.dimap.ufrn.br/csbc2011/anais/eventos/contents/WIM/WIM_Sessao_3_A rtigo_7_Seixas.pdf $>$. Acessado em Dezembro de 2011.

Shortliffe, E.H., Scott, A., Bischoff, M. (1981). "ONCOCIN: an expert system for oncology protocol management". Proc. Internat. Joint Conf. Artif. Intellig., p. 876881.

Skype. (2012). "Skype”. Disponível em: http://www.skype.com/>. Acessado em Janeiro de 2012.

Smart, J. F. (2011). “Jenkins: The Definitive Guide”. O’Reilly.

Soares, M. S. (2004). "Metodologias Ágeis Extreme Programming e Scrum para o Desenvolvimento de Software". Revista Eletrônica de Sistemas de Informação v. 3, n. 1.

Sociedade Brasileira de Pneumologia e Tisiologia. (2006). "IV Consenso Brasileiro no Manejo da Asma”. Disponibilizado no Congresso Brasileiro de Pneumologia.

Solé, D. et al. (1998). International study of asthma and allergies in childhood (ISAAC) written questionnaire: validation of the asthma component among Brazilian children. J Invest Allergol Clin Immunol 1998; 8: 376-82.

Sommerville, I. (2004). "Engenharia de Software”. São Paulo: Addison Wesley.

SonarSource. (2011) "Sonar". Disponível em: <http://www.sonarsource.com>. Acessado em Dezembro de 2011.

SpringSource. (2011). “Grails”. Disponível em: <http://grails.org/>. Acessado em Dezembro de 2011.

Team Viewer. (2012). "Team Viewer". Disponível em: $<$ http://www.teamviewer.com/pt/index.aspx > . Acessado em Janeiro de 2012.

Vital Wave Consulting (2009). "mHealth for Development: The Opportunity of Mobile Technology for Healthcare in the Developing World". United Nations Foundation, Vodafone Foundation. p. 9.

Yammer. (2012). "Yammer - The Enterprise Network". Disponível em: $<$ http://www.yammer.com>. Acessado em Janeiro de 2012. 